\title{
On the management of nanostructuring of electric spark coatings
}

\author{
V.I. Ivanov \\ tehnoinvest-vip@mail.ru \\ Federal Scientific Agroengineering Center VIM, $51^{\text {st }}$ Institutsky passage, Moscow, 109428, Russia
}

Analysis of the literature data indicates the great attention of specialists to the formation of ultrafine-grained (UFG) and nanocrystalline (NC) structures. Such structures are obtained by severe plastic deformation and other methods to improve the bulk properties of materials. Improving the properties of the surface layers of materials is achieved by modifying them with the formation of NC structures by different methods. In the article, in the development of earlier work, the results of studies of the formation of such structures by the method of electrospark alloying (ESD) are reflected. The aim of this work was to obtain experimentally quantitative data on the nanostructuring of electric-spark (ES) coatings, specifying the effect of the technological parameters of ESD and electrode material on this process. The studies included the determination of the energy parameters of the ESD process, changes in the mass of samples to obtain pulsed values, as well as the grain size of the structure of ES coatings. At the same time, modern certified technological and research equipment and recognized methods were used. The studies were carried out in a wide technological range: pulse energy $E=0.045 \ldots 0.29 \mathrm{~J}$, their duration $t_{\text {imp }}=20 \ldots 80 \mu$ s, frequency $f_{\text {imp }}=300 \ldots 1500 \mathrm{~Hz}$, the conditional value of the specific processing time $t_{\mathrm{sp}}=\tau \ldots . .5 \tau$. The result of the work is the following: coatings are obtained with a content of 5 to $50 \%$ of the granular structure of the nanoscale level using electrodes made of hard alloys VK8 $(92 \mathrm{WC}+8 \mathrm{Co})$ and STIM- $3 \mathrm{BOA}_{\mathrm{n}}\left(72 \mathrm{TiC}+17.5 \mathrm{Cr}_{3} \mathrm{C}_{2}+10 \mathrm{Ni}+0.5 \mathrm{Al}_{2} \mathrm{O}_{3}{ }^{\text {nano }}\right)$; it was found that an increase in the pulse energy leads to an increase in the grain size of the nanoscale level and a decrease in their fraction in the coating, and an increase in the pulse frequency and specific ESD time, on the contrary, to a decrease in the grain size and an increase in their number; the use of anode materials with a refractory nanocomponent also contributes to the nanostructuring of ES coatings. In developing this topic, attention is drawn to one of the important issues requiring separate studies - this is the study of the role and efficiency of pulses of the low-energy zone of electric regimes in the process of nanostructure of ES coatings.

Keywords: electrospark deposition, nanocrystalline structure, coating, grain, degree of nanostructuring.

УДК: 621.9 .048

\section{К вопросу управления наноструктурированием электроискровых покрытий}

\author{
Иванов В.И. \\ Федеральный научный агроинженерный центр ВИМ, 1-й Институтский проезд, 5, Москва, 109428, Россия
}

\begin{abstract}
Анализ литературных данных свидетельствует о большом внимании специалистов к формированию ультрамелкозернистых (УМЗ) и нанокристаллических (НК) структур. Такие структуры для улучшения объемных свойств материалов получают интенсивной пластической деформацией и другими методами. Улучшение свойств поверхностных слоев материалов достигается модификацией их с образованием НК структур разными методами. В статье, в развитие ранее выполненных работ, отражены результаты исследований формирования таких структур методом электроискрового легирования (ЭИЛ). Целью данной работы являлось получение экспериментальным путем количественных данных по наноструктурированию электроискровых (ЭИ) покрытий, конкретизирующих влияние технологических параметров ЭИЛ и материала электрода на этот процесс. Исследования включали определение энергетических параметров процесса ЭИЛ, изменения массы образцов с получением импульсных значений, а также величины зерен структуры ЭИ покрытий. Использованы при этом современное сертифицированное технологическое и исследовательское оборудование и признанные методики. Исследования выполнены в широком технологическом диапазоне: энергия импульсов $E=0.045 \ldots 0.29$ Дж, их длительность $t_{\text {имп }}=20 \ldots 80$ мкс, частота $f_{\text {имп }}=300 \ldots 1500$ Гц, условное значение удельного времени обработки $t_{\text {уд }}=\tau$...5 . Результатом работы является следующее: получены
\end{abstract}


покрытия с содержанием от 5 до 50\% зернистой структуры наноразмерного уровня при использовании электро-

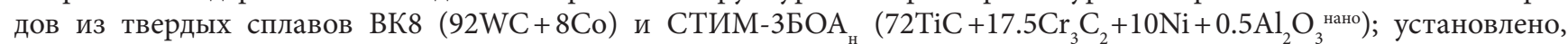
что повышение энергии импульсов ведет к увеличению размера зерен наноразмерного уровня и уменьшению их доли в покрытии, а повышение частоты импульсов и удельного времени ЭИЛ - наоборот, к уменьшению размера зерен и увеличению их количества; применение анодных материалов с тугоплавким нанокомпонентом также способствует наноструктуризации ЭИ покрытий. В развитие данной темы обращается внимание на один из важных вопросов, требующих отдельных исследований, — это изучение роли и эффективности импульсов низкоэнергетической зоны электрических режимов в процессе наноструктурирования ЭИ покрытий.

Ключевые слова: электроискровое легирование, нанокристаллическая структура, покрытие, зерно, степень наноструктурирования.

\section{1. Введение}

Условия эксплуатации современной техники требуют применения материалов и функциональных покрытий с улучшенными качественными характеристиками, а также эффективных технологий, обеспечивающих их получение. Наряду с созданием новых материалов и покрытий путем изменения химического и фазового составов, большое внимание уделяется формированию ультрамелкозернистых (УМЗ) и нанокристаллических (НК) структур [1,2]. Это в определенной степени обосновано известной зависимостью Холла-Петча $\sigma_{\mathrm{y}}=\sigma_{\mathrm{o}}+k_{\mathrm{y}} D^{-1 / 2}[3]$, устанавливающей связь предела текучести материала $\sigma_{y}$ которая влияет в прямой зависимости на его твердость, с размером зерен $D\left(\sigma_{\text {o }}-\right.$ внутреннее напряжение, препятствующее движению дислокаций; $k_{\mathrm{y}}-$ константа для каждого материала). Уточним, что эта зависимость наиболее выражена для металлических и керамических материалов с размером зерен более 1 мкм, но применяется с допущениями и для НК структур.

Формирование таких структур на металлических материалах достигается разными методами. Объемные свойства материалов улучшают созданием НК структур путем интенсивной пластической деформации. На основе работ П. Бриджмена [4], применившего кручение с осадкой тонких дисков, разработаны и используются многочисленные схемы интенсивного пластического деформирования металлов и сплавов: равноканальное угловое прессование, кручение под высоким давлением, всесторонняя ковка, циклическая деформация «осадкаэкструзия-осадка», винтовая экструзия и др.

Но улучшение эксплуатационных свойств различных изделий (детали, инструменты) достигается также модификацией их поверхностных слоев с образованием НК структур разными способами, включая лазерную, плазменную, химико-термическую обработку, ионную имплантацию и другие. Наряду с ними, отметим также метод электроискрового легирования (ЭИЛ), обеспечивающий создание при определенных условиях таких структур. Это было обнаружено еще на заре его применения: в работе [5] отмечено значительное наличие элементов размером порядка $600 \AA ̊$, т. е. 60 нм.

К настоящему времени накоплен положительный опыт в получении и использовании покрытий с НК структурой. Известны работы ряда российских (НИТУ МИСИС [6], ФНАЦ ВИМ [7], ОмГТУ [8], ИМ ХНЦ ДВО PAН [9]) и зарубежных научных центров (Украины [10], Германии и Польши [11]); ведутся успешные работы по ЭИЛ в других странах Европы, а также в США, Японии, Китае, Индии. Однако при наличии многих фактов получения и применения таких покрытий, экспериментальных данных об их свойствах, накопленном опыте получения для ЭИЛ новых анодных материалов с нанокомпонентом и их свойствах, мало исследованной областью остается управление наноструктурированием ЭИ покрытий энергомеханическими параметрами.

Ранее выполненными исследованиями [12] автором было установлено на основании полученных качественных характеристик, что метод ЭИЛ позволяет управлять дисперсностью кристаллической структуры покрытий, сформулированы основные принципы применения ЭИ процесса, способствующие формированию покрытий с НК и УМЗ структурой: применение электрических режимов с низкими энергией и длительностью импульсов; увеличение удельной длительности обработки; использование материалов катода с низкой теплопроводностью, а анода - низкой теплоемкостью; перенос элементов анода на катод в твердом состоянии.

Целью данной работы являлось получение экспериментальным путем количественных данных по наноструктурированию ЭИ покрытий, уточняющих влияние технологических параметров ЭИЛ и материала электрода на этот процесс.

\section{2. Программа и методика эксперимента}

Экспериментальные исследования включали определение энергетических параметров процесса ЭИЛ, изменения массы образцов (катод) и микроструктурные исследования покрытий. ЭИ покрытия нанесены на установке «БИГ-4» [13], энергетические исследования выполнены на комьютеризированном испытательном комплексе [14] согласно приведенной в этой работе методике, изменение массы определяли гравиметрическим методом с помощью аналитических весов «ВЛР-200», для электронной микроскопии использовали сканирующий зондовый микроскоп «SOLVER NEXT».

Определение зернистости структурных элементов покрытий выполняли измерениями с помощью указанного электронного микроскопа размера $d_{L}[\mathrm{Hм}]$ (условно - диаметр) наиболее мелких и крупных частиц, фиксируя этот диапазон для трех произвольно выбранных участков каждого образца и определяя средние значения. Также рассчитывали степень наноструктурирования $A$ или доли наноразмерных зерен в структуре покрытия как отношение суммарной их площади $F_{\text {гна }}$ 
к общей исследуемой площади $F$ согласно формуле $A=F_{\text {Гнано }} / F[\%]$.

Исследованиям подлежали модельные образцы из стали 45 (аналог сталям С45-Евросоюз, 1045 - США, 45 - Китай) в состоянии поставки с покрытиями, нанесенными анодными материалами из твердых сплавов ВК8 (92WC $+8 \mathrm{Co})$ и СТИМ-3БОА н $(72 \mathrm{TiC}+$ $\left.+17.5 \mathrm{Cr}_{3} \mathrm{C}_{2}+10 \mathrm{Ni}+0.5 \mathrm{Al}_{2} \mathrm{O}_{3}{ }^{\text {нано }}\right)$ по технологии согласно Табл. 1 .

Как видно из Табл. 1, принят для исследований широкий технологический диапазон нанесения покрытий: $E=0.045 \ldots 0.29$ Дж (кратность 6), $t_{\text {имп }}=20 \ldots 80$ мкс (кратность 4$), f_{\text {имп }}=300 \ldots 1500$ Гц (кратность 5$), t_{\text {уд }}=\tau \ldots 5 \tau$ (кратность 5).

Для нанесения покрытий использованы в качестве анода твердые сплавы, полученные порошковой металлургией ВК8 и методом самораспространяющегося высокотемпературного синтеза с добавкой нанокомпонента СТИМ-3БОА. Их выбор обусловлен значительным различием по физико-механическим свойствам, но общностью области применения и востребованностью для практического использования.

\section{3. Результаты и обсуждение}

Энергетические исследования процесса ЭИЛ в течение базового времени 10 с (принято для обеспечения достоверности экспериментальных данных [14]) показывают нестабильность искровых импульсов по величине энергии. Видно (Рис. 1), что импульсы сосредоточены в нижней, средней и верхней зонах. Верхняя зона образована наиболее мощными импульсами $(E=0.14-0.23$ Дж), условно назовем их «рабочими». Они вносят основной вклад в процесс формирования поверхностного слоя нового состава и структуры. Обычно ширина верхней зоны составляет около 30\% энергетической картины [14].

На Рис. 2 приведена для выбранных, различающихся по энергии импульсов, электрических режимов №№ 2,7 и 14 (Табл. 1) гистограмма, отражающая соотношение числа рабочих импульсов и количества всех зарегистрированных искровых импульсов. Доля рабочих импульсов составляет в данном случае от 34.7 до 49\%. Отметим здесь, что в общем случае их относительное количество может уменьшаться до 10\%, что показано более ранними

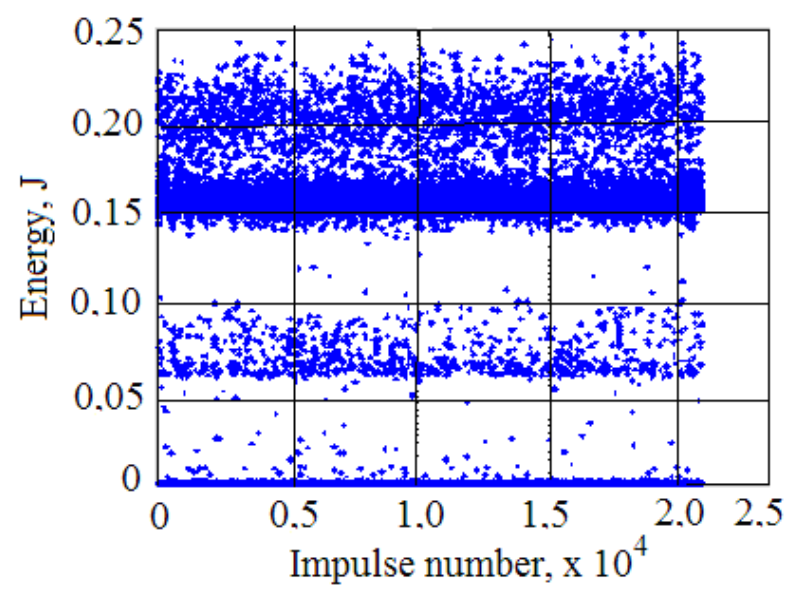

Рис. 1. Характерная энергетическая картина распределения искровых импульсов по величине энергии (базовое время - 10 c).

Fig. 1. The characteristic energy picture of the distribution of spark pulses by the amount of energy (base time $-10 \mathrm{~s}$ ).

Табл. 1. Технологические варианты ЭИ обработки образцов.

Table 1. Technological options for ES processing of samples.

\begin{tabular}{|c|c|c|c|c|c|c|}
\hline \multirow{2}{*}{$\begin{array}{l}\text { Вариант } \\
\text { Option }\end{array}$} & \multirow{2}{*}{$\begin{array}{l}\text { Электрод, размеры (мм) } \\
\text { Electrode, sizes (mm) }\end{array}$} & \multicolumn{3}{|c|}{$\begin{array}{l}\text { Электрический режим «БИГ-4» } \\
\text { Electric mode «BIG-4» }\end{array}$} & \multirow{2}{*}{$\begin{array}{l}t_{\text {общ }} / t_{\text {уд }} \\
t_{\text {tot }} / t_{\mathrm{sp}}\end{array}$} & \multirow{2}{*}{$\begin{array}{c}\text { Примечание } \\
\text { Note }\end{array}$} \\
\hline & & $\begin{array}{c}\text { № pежима* } \\
\text { Mode number }\end{array}$ & $\begin{array}{c}E(\text { Дж) } \\
E(\mathrm{~J}) \\
\end{array}$ & $\begin{array}{c}f_{\text {имп }}(\text { Цц }) / t_{\text {имп }}(\text { мКс }) \\
f_{\text {imp }}(\mathrm{Hz}) / t_{\text {imp }}(\mu \mathrm{s})\end{array}$ & & \\
\hline \multirow{2}{*}{\multicolumn{7}{|c|}{$\begin{array}{l}\text { Образец - сталь } 45 \text {, размеры } \varnothing 10 \times 4 \text { мм; площадь обработки } 0.8 \text { см}^{2} \\
\text { Sample }- \text { steel } 45 \text {, dimensions } \varnothing 10 \times 4 \mathrm{~mm} \text {; processing area } 0.8 \mathrm{~cm}^{2}\end{array}$}} \\
\hline & & & & & & \\
\hline 1 & \multirow{7}{*}{$\begin{array}{l}\text { BK8 / VK8 } \\
2.5 \times 5 \times 32\end{array}$} & $2(1-0.2)$ & 0.045 & $600 / 20$ & 1.44 & $2 \tau^{* *}$ \\
\hline 2 & & $7(2-0.4)$ & 0.09 & $600 / 40$ & 1.58 & $2 \tau$ \\
\hline 3 & & $14(3-0.8)$ & 0.29 & $600 / 80$ & 1.56 & $2 \tau$ \\
\hline 4 & & $7(2-0.4)$ & 0.09 & $600 / 40$ & 0.83 & $\tau$ \\
\hline 5 & & $7(2-0.4)$ & 0.09 & $600 / 40$ & 4.10 & $5 \tau$ \\
\hline 6 & & $6(2-0.2)$ & 0.09 & $300 / 40$ & 1.64 & $2 \tau$ \\
\hline 7 & & $10(2-1.0)$ & 0.09 & $1500 / 40$ & 1.44 & $2 \tau$ \\
\hline 8 & $\begin{array}{l}\text { СТИМ-БОА }{ }_{\text {н }} / \text { STIM-BOA }_{\mathrm{n}} \\
5 \times 5 \times 20\end{array}$ & $7(2-0.4)$ & 0.09 & $600 / 40$ & 1.66 & $2 \tau$ \\
\hline
\end{tabular}

* в скобках указаны номер режима и коэффициент энергии согласно паспорту установки.

** $\tau$ - условная величина минимального удельного времени ЭИЛ, достаточного для нанесения сплошного покрытия без пропусков, т. е. без участков с исходным рельефом поверхности.

Обозначения: $E$ - энергия импульсов (Дж); $f_{\text {имп }}$ - частота импульсов (Гц); $t_{\text {общ }}, t_{\text {уд }}-$ общее $($ с) и удельное (с/см²) время обработки. * in parentheses are the mode number and energy coefficient according to the installation certificate.

${ }^{* *} \tau$ is the conditional value of the minimum specific time of ESD, sufficient for applying a continuous coating without gaps, i. e. without areas with the original surface topography.

Designations: $E$ - pulse energy $(\mathrm{J}) ; f_{\mathrm{imp}}$ - pulse frequency $(\mathrm{Hz}) ; t_{\text {tot }}, t_{\mathrm{sp}}-$ total $(\mathrm{s})$ and specific $\left(\mathrm{s} / \mathrm{cm}^{2}\right)$ processing time. 


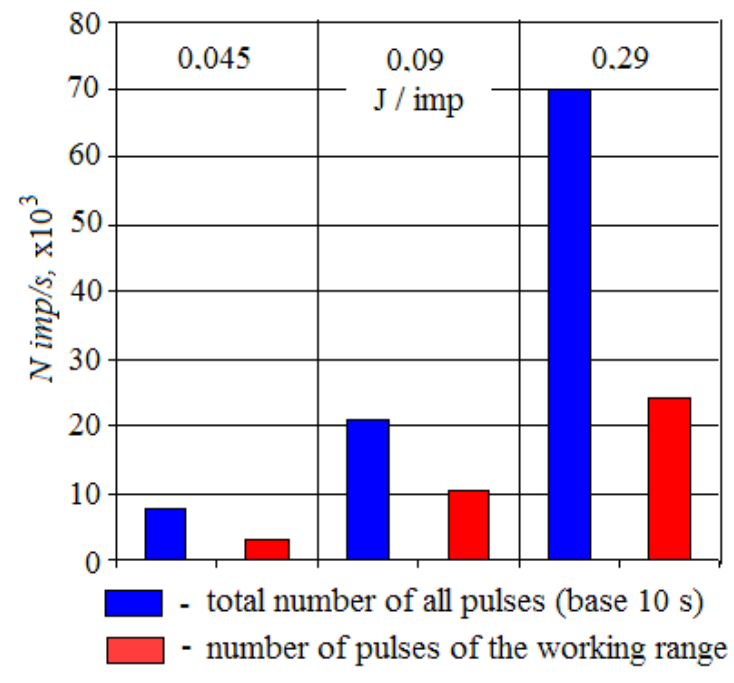

Puc. 2. (Color online) Соотношение общего количества и числа рабочих импульсов (установка «БИГ-4»).

Fig. 2. (Color online) The ratio of total and the number of working pulses (installation «BIG-4»).

исследованиями [12]. Очевидно, такое различие импульсов по энергии не может не отражаться на импульсной величине эрозии анодного материала и, соответственно, на размерах зерен структуры формируемого поверхностного слоя.

В результате ЭИ обработки образцов увеличилась их масса. Это видно из данных Табл. S1 (дополнительный материал), где приведены значения общего прироста массы $\Delta m_{k}$, а также его импульсные значения $\Delta m_{k \text { имп }}$. Из анализа этих данных следует, что рассматриваемые технологические параметры в разной степени влияют на изменение массы образца, как одного из определяющих критериев формирования ЭИ покрытия. Ранжируя управляемые параметры в порядке убывания на основе использования импульсных значений прироста массы образцов, отметим, в первую очередь, энергию импульсов, затем их частоту и потом удельное время.

Выполненные исследования покрытий методом электронной микроскопии позволили установить влияние технологических параметров ЭИЛ и материала электрода также на зернистость их структуры. На Рис. 3 приведены фотографии микрошлифов, по которым видно различие по размерам элементов структуры в виде зерен. Результаты обработки полученных снимков исследуемых покрытий отражены в Табл. S1 (дополнительный материал) и на Рис. 4.

Согласно полученным данным видно большое влияние энергии импульсов на размер зерен наноразмерного уровня - в прямой зависимости - и в обратной зависимости - на их долю в покрытии. Иным образом и одинаковой направленности влияет на эти характеристики изменение частоты импульсов и удельного времени ЭИЛ: повышение значений этих параметров ведет к уменьшению размера зерен и увеличению их количества. Таким же образом, как частота импульсов и удельное время ЭИЛ, оказывает влияние на исследуемые характеристики применение анодных материалов с тугоплавким нанокомпонентом. Это видно из сравнения результатов, полученных при использовании электродов из сплавов ВК8, у которых исходная величина карбидных зерен составляет 1-3 мкм, и СТИМ-3БОА.

Также отметим корреляцию изменения импульсных значений прироста массы катода и размеров зерен с изменением энергии и частоты импульсов. Видна тенденция уменьшения величины зерна и увеличения их количества со снижением энергии импульсов. Из этого следует вывод о целесообразности проведения дополнительных исследований по изучению роли импульсов низкоэнергетического диапазона, которые на данном этапе не учитываются, в процессе формирования ЭИ покрытий, их структуры и свойств.

\section{4. Заключение}

Выполненные комплексные экспериментальные исследования обеспечили получение количественных данных по наноструктурированию ЭИ покрытий, что уточняет и дополняет сведения о влиянии технологических параметров ЭИЛ и материала электрода на этот процесс. В исследованном технологическом диапазоне при использовании электродов из твердых сплавов ВК8 и СТИМ-3БОА н получены покрытия с содержанием

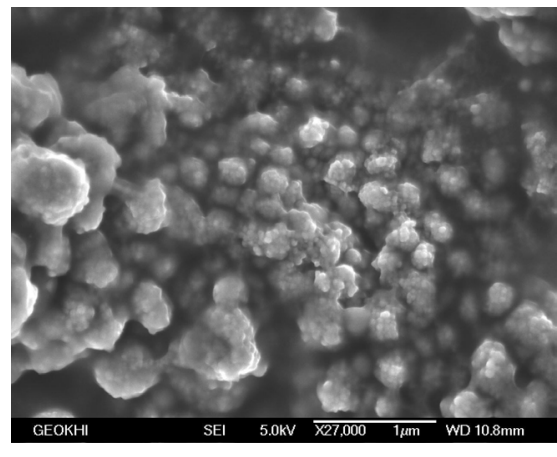

a

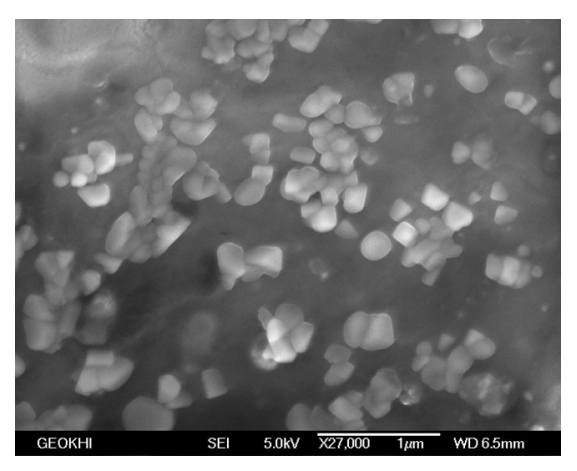

b

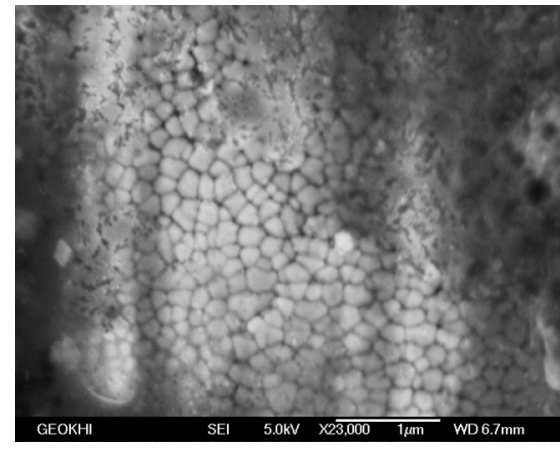

C

Рис. 3. Фотографии структуры ЭИ покрытий на стали 45, нанесенных на установке «БИГ-4» электродами ВК8 (a,b) и СТИМ-3БОА

Fig. 3. Photos of the structure of ES coatings on steel 45 , deposited on the BIG-4 installation with VK8 (a, b) and STIM-3BOAn (c) electrodes $E=0.09 \mathrm{~J}, f_{\mathrm{imp}}=600 \mathrm{~Hz}, 2 \tau(\mathrm{a}, \mathrm{c}) ; E=0.29 \mathrm{~J}, f_{\mathrm{imp}}=600 \mathrm{~Hz}, 2 \tau(\mathrm{b})$. 


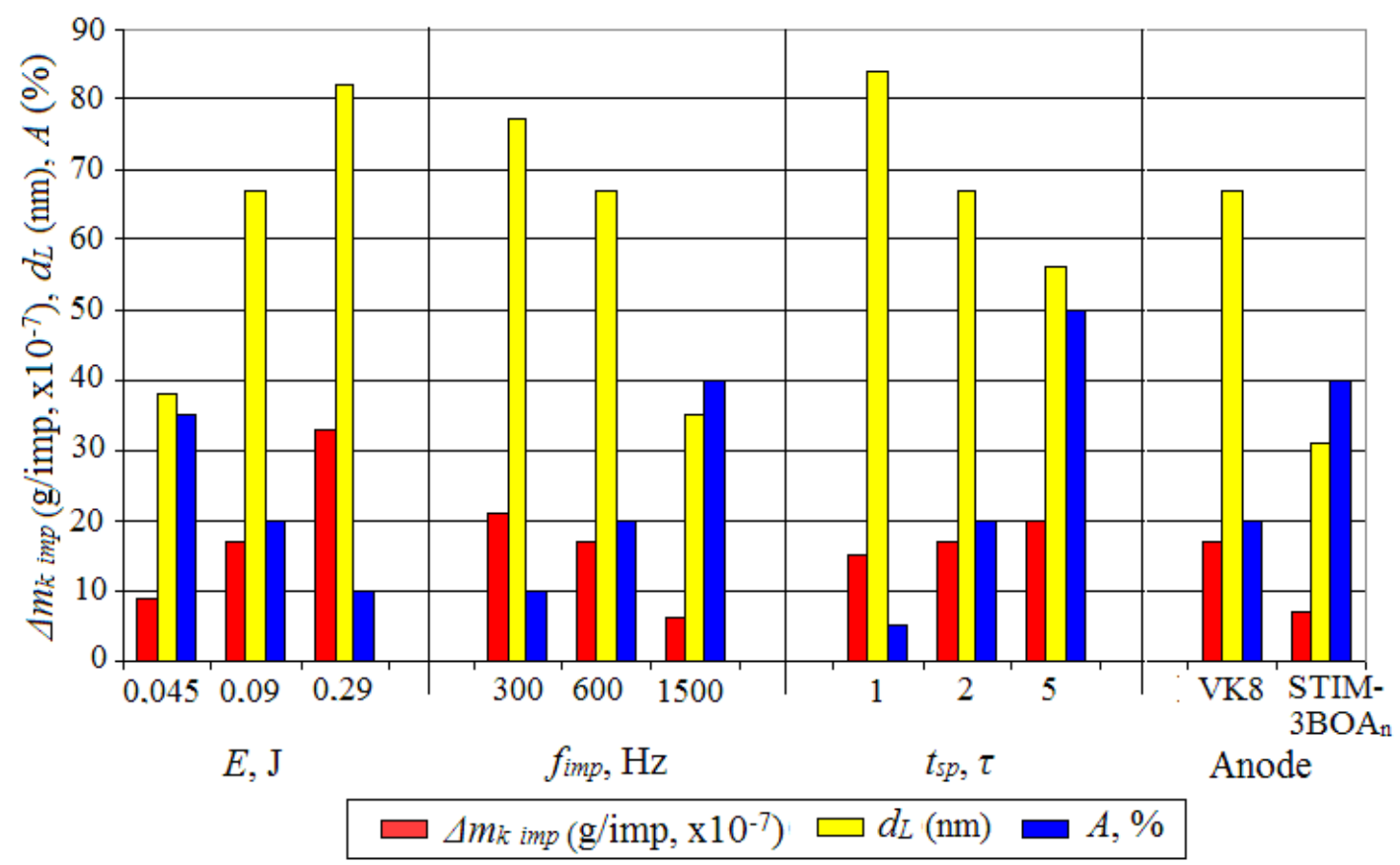

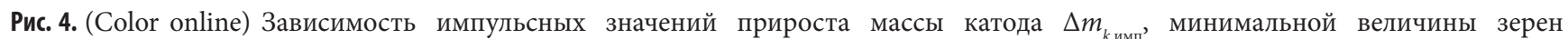
наноразмерного уровня $d_{L}$ и их доли $A$ в покрытии от технологических параметров ЭИЛ и материала электрода.

Fig. 4. (Color online) The dependence of the pulse values of the increase in cathode mass $\Delta m_{k \text { imp }}$, the minimum size of the grains of the nanoscale level $d_{L}$ and their fraction $A$ in the coating from technological parameters of ESD and electrode material.

от 5 до $50 \%$ зернистой структуры наноразмерного уровня. При этом установлено, что повышение энергии импульсов ведет к увеличению размера зерен наноразмерного уровня и уменьшению их доли в покрытии, а повышение частоты импульсов и удельного времени ЭИЛ - наоборот, к уменьшению размера зерен и увеличению их количества. Применение анодных материалов с тугоплавким нанокомпонентом также способствует наноструктуризации ЭИ покрытий. Требует отдельных исследований установление роли импульсов низкоэнергетической зоны электрических режимов, их влияния на процесс формирования измененного поверхностного слоя, его структуры и свойств.

Дополнительный материал / Supplementary material. Электронная версия статьи содержит дополнительный материал (таблиия, включающая сведения об условиях обработки образиов методом ЭИЛ и результать исследования изменения массы образиов и величины зерен наноразмерного уровня полученных покрытий), доступный безвозмездно на сайте журнала (lettersonmaterials. com). I The online version of this paper contains supplementary material (a table containing information on the conditions for processing samples by the ESD method and the results of studying changes in the mass of samples and the size of grains of the nanoscale level of the obtained coatings) available free of charge at the journal's Web site (lettersonmaterials.com).

Благодарности / Acknowledgments. Автор благодарен к. т. н. С. Д. Карпухину - доценту кафедры МТ-8 «Материаловедение» МГТУ им. Н.Э. Баумана - за выполненные исследования покрытий методом электронной микроско- nuu. / The author is grateful to Ph.D. S.D. Karpukhin Associate Professor of the Department of MT-8 "Materials Science" MSTU N.E. Bauman - for the performed research of coatings by electron microscopy.

\section{Литература/References}

1. A.S. Edelstein, R.C. Cammarata. Nanomaterials: Synthesis, Properties and Applications. Baltimor, The Johns Hopkins University (1998) 620 p.

2. A.I. Gusev. Nanomaterials, nanostructures, nanotechnology. 2rd edn., Rev. Moscow, Fizmatlit (2009) 416 p. (in Russian) [А.И. Гусев. Наноматериалы, наноструктуры, нанотехнологии. 2-е изд., испр. Москва, Физматлит (2009) 416 с.]

3. N. J. Petch. J. Iron and Steel Inds. 174, 25 (1953).

4. P. Bridgman. The physics of high pressure. London, G. Bell and sons, Itd. (1931).

5. L.S. Palatnik. Izv. USSR Academy of Sciences. - Ser. Physical. 15 (1), 80 (1951). (in Russian) [Л. С. Палатник. Изв. АН СССР. - Сер. физ. 15 (1), 80 (1951).]

6. E.A. Levashov, A.E. Kudryashov, O.V. Malochkin, S. A. Glukhov, T. A. Sviridova, F. Gammel, R. Suchentrunk. Russian Journal of Non-Ferrous Metals. 42 (5), 27 (2001).

7. F. Kh. Burumkulov, P. V. Senin, S. A. Velichko, V. I. Ivanov, P. A. Ionov, M. A. Okin. Surface Engineering and Applied Electrochemistry. 45 (6), 455 (2009). rossref

8. D. N. Korotaev. Technological possibilities of forming wear-resistant nanostructures by electrospark alloying: monograph. Omsk, SibADI (2009) 255 p. (in Russian) [Д.Н. Коротаев. Технологические возможности формирования износостойких наноструктур 
электроискровым легированием: монография. Омск, СибАДИ (2009) 255 с.]

9. S. V. Nikolenko, A.P. Kuzmenko, D.I. Timakov, P.V. Abakymov. Surface Engineering and Applied Electrochemistry. 47 (3), 217 (2011). Crossref

10. V. Martsynkovskyy, V. Tarelnyk, E. Konoplianchenko, O. Gaponova. Microstructure and Properties of Microand Nanoscale Materials, Films, and Coatings (NAP 2019). Springer, Singapore (2019) 135 p. Crossref

11. N. Radek, K. Bartkowiak. Physics Procedia. 39, 295 (2012). Crossref

12. V.I. Ivanov. Hardening, coating and repair technologies: theory and practice: international materials. scientificpractical conf. St. Petersburg, Polytechnic Publishing House. University (2016) p. 164. (in Russian)
[В.И. Иванов Технологии упрочнения, нанесения покрытий и ремонта: теория и практика: материалы междунар. науч.-практ. конф. Санкт-Петербург, Издво политехн. ун-та (2016) с. 164.]

13. V. I. Ivanov, F. Kh. Burumkulov, P. V. Senin, S. A. Velichko, P. A. Ionov, N. V. Rakov. Guidelines for the use of electric spark installation «BIG-4». Saransk, Mordov Publishing House University (2011) 35 p. (in Russian) [В. И. Иванов, Ф.Х. Бурумкулов, П.В. Сенин, С.А. Величко, П.А. Ионов, Н.В. Раков. Методические указания по применению электроискровой установки «БИГ-4». Саранск, Изд-во Мордов. ун-та (2011) 35 с.]

14. V.I. Ivanov. Electronic processing of materials. 51 (1), 105 (2015). (in Russian) [В.И. Иванов. Электронная обработка материалов. 51 (1), 105 (2015).] 